Pacific

Journal of

Mathematics

THE SELF-SIMILAR PROFILES OF GENERALIZED KPZ EQUATION

YUANWEI QI AND MingXin WANG

Volume $201 \quad$ No. 1

November 2001 


\title{
THE SELF-SIMILAR PROFILES OF GENERALIZED KPZ EQUATION
}

\author{
YUANWEI QI AND MingXiN WANG
}

\begin{abstract}
In this paper we consider, for $1 \leq m<p<2$, the generalized KPZ equation $u_{t}=\Delta\left(u^{m}\right)-|\nabla u|^{p}$. For $m=1$, we show existence and uniqueness of the so called very singular solution which is self-similar. A complete classification of self-similar solutions is also given. For $m>1$, we establish the existence of very singular self-similar solution and prove that such a solution must have compact support. Moreover, we derive the interface relation. Recent experience with parallel equations where the gradient term $|\nabla u|^{p}$ is replaced by $u^{p}$ indicates that the self-similar solutions are crucially important in study intermediate asymptotic behavior of general solutions.
\end{abstract}

\section{Introduction.}

In this paper, we consider the equation

$$
u_{t}=\Delta u-|\nabla u|^{p} \quad \text { in } \quad R^{n} \times(0,+\infty), \quad 1<p<2
$$

and its porous media counterpart

$$
u_{t}=\Delta\left(u^{m}\right)-|\nabla u|^{p} \quad \text { in } \quad R^{n} \times(0,+\infty), \quad 1<m<p<2 .
$$

Equation (1.1) is called generalized KPZ equation which arises from modelling of growth mechanism for surfaces through ballistic deposition, see [15], [16]. The model is derived from consideration that the growth mechanism is governed (approximating) by local rules. In such a model, $u(x, t)$ is the height above the underlying substrate which describes the interface profile, or the surface of the material. Experiments and numerical simulation show that $u(x, t)$ behaviours in a self-similar way. One important aspect in the study of such a model is then to find out the scaling exponents and functions which characterize the self-similarity of surface on a large space-time scale.

The actual physical model involving (1.1) is subject to random initial data and thorough analytical understanding is beyond our ability.

In this paper, we consider a simpler case, where the initial value $u(x, 0)=$ $u_{0}(x)$ is a deterministic function. 
The main purpose is to study the existence and detailed characterization of special profiles of various type of self-similar solutions to (1.1) and (1.2), see Theorems 2.1 and 3.1 for more details.

Here by a self-similar solution we mean that $u$ has the form

$$
u(x, t)=t^{-\alpha} f\left(|x| t^{-\beta}\right) .
$$

For Equation (1.1),

$$
\alpha:=\frac{2-p}{2(p-1)}, \quad \beta:=\frac{1}{2},
$$

and $f$ as a function of $r=|x| t^{-\beta}$, defined on $[0,+\infty)$, solves

$$
f^{\prime \prime}+\frac{n-1}{r} f^{\prime}+\beta r f^{\prime}+\alpha f-\left|f^{\prime}\right|^{p}=0 \quad \forall r>0 .
$$

For Equation (1.2),

$$
\begin{aligned}
\alpha & :=\frac{2-p}{p(3-m)-2}, \\
\beta & :=\frac{p-m}{p(3-m)-2} \quad(1<m<p<2 \Rightarrow p(3-m)-2>0),
\end{aligned}
$$

and $f$ as a function of $r=|x| t^{-\beta}$, defined on $[0,+\infty)$, solves

$$
\left(f^{m}\right)^{\prime \prime}+\frac{n-1}{r}\left(f^{m}\right)^{\prime}+\beta r f^{\prime}+\alpha f-\left|f^{\prime}\right|^{p}=0 \quad \forall r>0 .
$$

In particular, we are able to show the existence of the so called very singular solutions for both (1.1) and (1.2).

By a singular solution we mean a nonnegative and nontrivial solution which is continuous in $R^{n} \times[0,+\infty) \backslash\{(0,0)\}$ and satisfies

$$
\lim _{t \searrow 0} \sup _{|x|>\varepsilon} u(x, t)=0 \quad \forall \varepsilon>0 .
$$

A singular solution is called a very singular solution if

$$
\lim _{t \searrow 0} \int_{|x| \leq \varepsilon} u(x, t) d x=\infty \quad \forall \varepsilon>0 .
$$

Note that condition (1.9) is equivalent to, if $u$ is given by (1.3),

$$
\lim _{r \rightarrow \infty} r^{\alpha / \beta} f(r)=0
$$

Furthermore, if $n \beta<\alpha$ and the solution $f$ of (1.5) or (1.7) satisfies (1.10), then $u(x, t)$ given explicitly by (1.3) satisfies (1.8) and (1.9), i.e., it is a very singular self-similar solution of (1.1) or (1.2). 
In recent years, several authors of [3]-[9], [11]-[14] and [17]-[20] studied the existence of very singular self-similar solutions and their role in describing the intermediate asymptotic behavior of general solutions to

$$
u_{t}=\triangle\left(u^{m}\right)-u^{p}, \quad 0<m<\infty, \quad p>1
$$

and

$$
u_{t}=\operatorname{div}\left(|\nabla u|^{m-1} \nabla u\right)-u^{p}, \quad 0<m<\infty, \quad p>1 .
$$

But, it can be seen from what follows that (1.1) and (1.2) have some peculiar properties very different from (1.11) and (1.12).

We mention that the Cauchy problem of (1.1) and related problems have been studied by Ben-Artzi and Koch [1], B. Gilding et al [10] and more recently by Benachou and Laurencot [2]. In particular, Benachou and Laurencot [2] proved the existence and uniqueness of very singular solution of (1.1), but their proof is different from ours.

The organization of this paper is as follows. In $\S 2$ we study (1.5) and give a complete classification of its solutions. In particular, the existence and uniqueness of very singular self-similar solution is proved. In $\S 3$, we study (1.7) and establish the existence of a very singular self-similar solution which has compact support. The interface relation is also shown.

\section{Study of self-similar solutions to (1.1).}

In this section we study (1.5) and give a complete classification of its solutions in relation to the initial value $f(0)$. In particular, we prove the existence and uniqueness of very singular self-similar solution. We consider the solution of (1.5) with initial value

$$
f(0)=a>0, \quad f^{\prime}(0)=0 .
$$

For each $a>0,(1.5)$ and $(2.1)$ has a unique solution $f(r ; a)$, at least locally. If we multiply (1.5) by $r^{n-1}$ and integrate from 0 to $r$, we get

$$
f^{\prime} r^{n-1}=-\beta r^{n} f(r)+\int_{0}^{r} s^{n-1}\left[(\beta n-\alpha) f+\left|f^{\prime}\right|^{p}\right] d s .
$$

Another integration on $[0, r]$ after dividing the above equation by $r^{n-1}$ and simple calculation yield

$$
f(r)=a-a \frac{\alpha}{2 n} r^{2}+o\left(r^{2}\right) .
$$

This shows how the solution behaviours as $r \rightarrow 0$.

If we denote by $(0, R(a))$ the maximal existence interval where $f>0$, then $f^{\prime}<0$ in $(0, R(a))$ and either (i) $R(a)=\infty$ and $\lim _{r}>\infty f(r ; a)=0$, or (ii) $R(a)<\infty$ and $f(R(a) ; a)=0$. The main results of this section read as follows. 
Theorem 2.1. Assume that $1<p<2$. For each $a>0$, let $f(r ; a)$ be the solution of (1.5), (2.1). Then the following conclusions hold:

(i) If $2 \alpha \leq n$, then $R(a)=\infty$ and $\liminf _{r \rightarrow \infty} r^{2 \alpha} f(r ; a)>0$.

(ii) If $2 \alpha>n$, then there exists $a^{*}>0$ such that the following classification is valid:

(a) If $a \in\left(0, a^{*}\right)$, then $R(a)<\infty$ and $f^{\prime}<0$ in $(0, R(a)$ ].

(b) If $a \in\left(a^{*}, \infty\right)$, then $R(a)=\infty, f(r ; a)$ is strictly increasing and $f^{\prime}(r ; a)$ is strictly decreasing with respect to a. And for some $k(a)>$ 0 ,

$$
\begin{array}{r}
f(r ; a)=k(a) r^{-2 \alpha}\left\{1+2 \alpha\left(2+2 \alpha-n-[2 \alpha k(a)]^{p-1}\right) r^{-2}+o\left(r^{-2}\right)\right\} \\
\text { as } r \rightarrow \infty .
\end{array}
$$

(c) If $a=a^{*}$, then $\lim _{r \rightarrow \infty} r^{2 \alpha} f\left(r ; a^{*}\right)=0$, and for some $k\left(a^{*}\right)>0$,

$$
\begin{array}{r}
f\left(r ; a^{*}\right)=k\left(a^{*}\right) r^{2 \alpha-n} e^{-r^{2} / 4}\left\{1-2(2 \alpha-n)(\alpha-1) r^{-2}+o\left(r^{-2}\right)\right\} \\
\text { as } r \rightarrow \infty .
\end{array}
$$

This theorem shows that (1.1) has a very singular self-similar solution if and only if $1<p<(2+n) /(1+n)$, and in case of existence the solution is unique.

We write (1.5) as

$$
\left\{\begin{array}{l}
f^{\prime}=v, \\
v^{\prime}=-\frac{n-1}{r} v-\frac{r}{2} v-\alpha f-|v|^{p-1} v .
\end{array}\right.
$$

Lemma 2.1. Assume that $a>0$ and $f=f(r ; a)$ is the solution of (1.5), (2.1), $(0, R(a))$ is the maximal existence interval where $f>0$. Then

$$
\left|f^{\prime}(r)\right| \leq(\alpha a)^{1 / p} \quad \text { for all } \quad 0 \leq r \leq R(a) .
$$

Proof. First, we consider the case where $f^{\prime \prime}$ is negative in an interval. If there exist two constants $b$ and $c$ such that $0<b<c \leq R(a)$ and $f^{\prime \prime}(r) \leq 0$ in $(b, c)$. Then by Equation (1.5) we have $\left|f^{\prime}(r)\right|^{p} \leq \alpha f(r) \leq \alpha a$. Therefore, $\left|f^{\prime}(r)\right| \leq(\alpha a)^{1 / p}$ for all $r \in[b, c]$.

On the other hand, if $b_{1}$ and $c_{1}$ are so given that $0<b_{1}<c_{1} \leq$ $R(a), \quad f^{\prime \prime}(r) \geq 0$ in $\left(b_{1}, c_{1}\right)$ and $f^{\prime \prime}\left(b_{1}\right)=0$, then $f^{\prime}(r) \geq f^{\prime}\left(b_{1}\right)$, and hence $\left|f^{\prime}(r)\right| \leq\left|f^{\prime}\left(b_{1}\right)\right|$ for all $r \in\left[b_{1}, c_{1}\right]$.

Since $f^{\prime \prime}(0)=-\alpha a / n<0$, the above consideration show that the conclusion of Lemma 2.1 holds.

For any given $\lambda>0$, we denote $\mathcal{L}_{\lambda}=\{(f, v): f>0,-\lambda f<v<0\}$.

Lemma 2.2. For any given $\lambda>0$ there exists an $r_{\lambda}:=2(\lambda+\alpha / \lambda)$ such that $\mathcal{L}_{\lambda}$ is positively invariant for $r>r_{\lambda}$. That is, if $\left(f\left(r_{\lambda}\right), v\left(r_{\lambda}\right)\right) \in \mathcal{L}_{\lambda}$, then the orbit $(f(r), v(r))$ of (2.4) remains in $\mathcal{L}_{\lambda}$ for all $r \geq r_{\lambda}$. 
Proof. Since the vector field points into $\mathcal{L}_{\lambda}$ from the positive $f$-axis, we need to show that it also points into $\mathcal{L}_{\lambda}$ from the ray

$$
l_{\lambda}=\{(f, v): f>0, \quad v=-\lambda f\} .
$$

We have on $l_{\lambda}$,

$$
\frac{v^{\prime}}{f^{\prime}}=-\frac{n-1}{r}-\frac{r}{2}-\alpha \frac{f}{v}-|v|^{p-1}<-\frac{r}{2}+\frac{\alpha}{\lambda} .
$$

Hence,

$$
\frac{v^{\prime}}{f^{\prime}}<-\lambda \quad \text { on } l_{\lambda}
$$

if $r \geq r_{\lambda}:=2(\lambda+\alpha / \lambda)$. This completes the proof.

By using similar arguments as those of Lemmas 5-7 in [4], the following result can easily be shown to hold. But, for simplicity, we omit the details.

Lemma 2.3. Suppose $f(r ; a)>0$ for all $r>0$. Then

(i) $(f(r ; a), v(r ; a)) \rightarrow(0,0) \quad$ as $r \rightarrow \infty$.

(ii) $\lim _{r \rightarrow \infty} \frac{v(r ; a)}{f(r ; a)}=L_{a}$ exists, and $L_{a}=0$ or $L_{a}=-\infty$.

We now prove Theorem 2.1(i), which gives the nonexistence results of very singular self-similar solutions when $2 \alpha \leq n$.

Proof of Theorem 2.1(i). Suppose $2 \alpha \leq n$. Multiplying (1.5) by $r^{2 \alpha-1}$ we have, for $r \in(0, R(a))$,

$$
\left(r^{2 \alpha-1} f^{\prime}+\frac{1}{2} r^{2 \alpha} f\right)^{\prime}=(2 \alpha-n) r^{2 \alpha-2} f^{\prime}+r^{2 \alpha-1}\left|f^{\prime}\right|^{p}>0 .
$$

The function $g(r):=r^{2 \alpha-1} f^{\prime}+\frac{1}{2} r^{2 \alpha} f$ is strictly increasing in $(0, R(a))$. Note that $\lim _{r \backslash 0} g(r)=0$, we get $g>0$ in $(0, R(a))$. Since $f^{\prime}<0$, we conclude that $R(a)=\infty$ and $f \searrow 0$ as $r \nearrow \infty$. In addition, $r^{2 \alpha} f(r ; a) \geq 2 g(r)$ and $g(r)$ is increasing, hence $\liminf _{r \rightarrow \infty} r^{2 \alpha} f>0$. This completes the proof.

In the sequel of this section we always assume that $2 \alpha>n$. Let $L_{a}$ be given as in Lemma 2.3, we define

$$
\begin{aligned}
& \mathcal{A}=\{a>0: R(a)<\infty\}, \\
& \mathcal{B}=\left\{a>0: R(a)=\infty \text { and } L_{a}=0\right\}, \\
& \mathcal{C}=\left\{a>0: R(a)=\infty \text { and } L_{a}=-\infty\right\} .
\end{aligned}
$$

By Lemma 2.3 we know that $\mathcal{A} \cup \mathcal{B} \cup \mathcal{C}=(0, \infty)$. It is obvious that these sets are disjoint.

Lemma 2.4. The set $\mathcal{A}$ is nonempty and open. 
Proof. We choose $a=\epsilon \ll 1$ and let

$$
w_{\epsilon}(r)=\varepsilon^{-1} f(r ; \varepsilon) \text {. }
$$

Then $w_{\varepsilon}$ satisfies

$$
\left\{\begin{array}{l}
w_{\varepsilon}^{\prime \prime}+\frac{n-1}{r} w_{\varepsilon}^{\prime}+\frac{r}{2} w_{\varepsilon}^{\prime}+\alpha w_{\varepsilon}+\varepsilon^{p-1}\left|w_{\varepsilon}^{\prime}\right|^{p-1} w_{\varepsilon}^{\prime}=0 \\
w_{\varepsilon}(0)=1, \quad w_{\varepsilon}^{\prime}(0)=0
\end{array}\right.
$$

Let $E\left(w_{\varepsilon}\right)=\alpha w_{\varepsilon}^{2}+\left(w_{\varepsilon}^{\prime}\right)^{2}$, then $\frac{d}{d r} E\left(w_{\varepsilon}\right) \leq 0$. Therefore, $E\left(w_{\varepsilon}\right) \leq \alpha$ for all $\varepsilon>0$. Consequently, both $w_{\varepsilon}$ and $w_{\varepsilon}^{\prime}$ are uniformly bounded with respect to $r \geq 0$ and $\varepsilon>0$. It follows by the standard continuity argument that

$$
w_{\varepsilon} \rightarrow w \quad \text { as } \varepsilon \rightarrow 0 \text { in } C^{2}([0, R])
$$

for any $R>0$, where $w$ is the solution of the reduced problem

$$
\left\{\begin{array}{l}
w^{\prime \prime}+\frac{n-1}{r} w^{\prime}+\frac{r}{2} w^{\prime}+\alpha w=0 \\
w(0)=1, \quad w^{\prime}(0)=0
\end{array}\right.
$$

We claim that $w$ has a zero. Suppose on the contrary that $w(r)>0$ for all $r>0$. By (2.6) we have

$$
\left(r^{n-1} w^{\prime}(r)+\frac{1}{2} r^{n} w(r)\right)^{\prime}=\left(\frac{n}{2}-\alpha\right) r^{n-1} w(r)<0 .
$$

Therefore, $w^{\prime}(r)+\frac{1}{2} r w(r)<0$ for all $r>0$. Thus we have $w(r) \leq \exp \left\{-\frac{r^{2}}{2}\right\}$, and $\lim \sup _{r \rightarrow \infty} r^{n-1} w^{\prime}(r)=0$. But, an integration of (2.7) gives

$$
r^{n-1} w^{\prime}(r)+\frac{1}{2} r^{n} w(r)=\int_{0}^{r}\left(\frac{n}{2}-\alpha\right) s^{n-1} w(s) d s<-C, \quad r \gg 1
$$

for some constant $C>0$. It is a contradiction.

Since $w^{\prime}<0$ at the first zero of $w$, it follows that for $\varepsilon$ sufficiently small, $w_{\varepsilon}$ has a zero as well. This shows that $\mathcal{A}$ is nonempty.

By the uniqueness and continuous dependence on the initial data of solution we see that $\mathcal{A}$ is open.

Lemma 2.5. The set $\mathcal{B}$ is nonempty and open.

Proof. We first show that if initial data $a$ is suitably large then the corresponding orbit must stay in $\mathcal{L}_{1}$ for all $r \geq 0$. This implies that $a \in \mathcal{B}$.

Let $r_{0}$ be the first value such that the orbit intersects with the boundary of $\mathcal{L}_{1}$. It is clear that $v\left(r_{0}\right)=-f\left(r_{0}\right)$. Consequently, using Lemma 2.1, we have

$$
f\left(r_{0}\right)=-v\left(r_{0}\right) \leq(\alpha a)^{1 / p}
$$


and

$$
\begin{aligned}
f\left(r_{0}\right) & =f(0)+\int_{0}^{r_{0}} f^{\prime}(s) d s \\
& \geq a+\int_{0}^{r_{0}}\left\{-(\alpha a)^{1 / p}\right\} d s \\
& =a-(\alpha a)^{1 / p} r_{0} .
\end{aligned}
$$

(2.8) combined with (2.9) yields

$$
r_{0} \geq \frac{a-(\alpha a)^{1 / p}}{(\alpha a)^{1 / p}} \rightarrow \infty \quad \text { as } a \rightarrow \infty .
$$

It contradicts to Lemma 2.2.

Now we prove that $\mathcal{B}$ is open. Suppose $a_{0} \in \mathcal{B}$. Then by the definition of $\mathcal{B}$, there exists $r_{0}>0$ such that $\left(f\left(r_{0} ; a_{0}\right), v\left(r_{0} ; a_{0}\right)\right) \in \mathcal{L}_{1}$. Hence, by continuous dependence on initial data there exists a neighbourhood $\Sigma$ of $a_{0}$ such that if $a \in \Sigma$, then $f(r ; a)>0$ for all $r \in\left[0, r_{0}\right]$ and $\left(f\left(r_{0} ; a\right), v\left(r_{0} ; a\right)\right) \in$ $\mathcal{L}_{1}$. It follows from Lemmas 2.2 and 2.3 that if $a \in \Sigma$, the corresponding $L_{a}=0$, so that $a \in \mathcal{B}$.

Lemma 2.6. Assume that $f_{1}(0)=a_{1}>0, \quad f_{2}(0)=a_{2}>0$. If $a_{2}>a_{1}$ then

$$
f_{2}(r)>f_{1}(r), \quad f_{2}^{\prime}(r)<f_{1}^{\prime}(r) \quad \forall 0<r<R\left(a_{1}\right) .
$$

Proof. Let $w=f_{1} f_{2}^{\prime}-f_{1}^{\prime} f_{2}$, then $w$ satisfies

$$
\begin{gathered}
w^{\prime}+\left\{\frac{n-1}{r}+\frac{r}{2}+\left|f_{2}^{\prime}\right|^{p-1}\right\} w \\
=-f_{1}^{\prime} f_{2}\left[\left|f_{2}^{\prime}\right|^{p-1}-\left|f_{1}^{\prime}\right|^{p-1}\right] \\
\triangleq F(r) .
\end{gathered}
$$

Because $f_{i}^{\prime \prime}(0)=-a_{i} / n, a_{1}<a_{2}$ and $f_{i}^{\prime}(0)=0$, it follows that $f_{2}^{\prime}(r)<f_{1}^{\prime}(r)$, and consequently $F(r)>0$ for $r \ll 1$. Denote

$$
r_{0}=\sup \left\{0<r<R\left(a_{1}\right): f_{2}^{\prime}(s)<f_{1}^{\prime}(s) \forall s \in(0, r)\right\} .
$$

Then we have

$$
w^{\prime}+\left\{\frac{n-1}{r}+\frac{r}{2}+\left|f_{2}^{\prime}\right|^{p-1}\right\} w=F(r)>0 \quad \forall 0<r<r_{0} .
$$

Since $w(0)=0$, it follows that

$$
w(r)>0 \text {, i.e., }\left(f_{2} / f_{1}\right)^{\prime}>0 \quad \forall 0<r<r_{0} .
$$

Therefore, $f_{2}(r)>f_{1}(r)$ for all $0 \leq r \leq r_{0}$. 
We assert that $r_{0}=R\left(a_{1}\right)$. Suppose to the contrary that $r_{0}<R\left(a_{1}\right)$, then $f_{2}^{\prime}\left(r_{0}\right)=f_{1}^{\prime}\left(r_{0}\right)$. On the other hand, by (1.5) we have

$$
\begin{aligned}
f_{2}^{\prime \prime}\left(r_{0}\right) & =-\frac{n-1}{r_{0}} f_{2}^{\prime}\left(r_{0}\right)-\frac{r_{0}}{2} f_{2}^{\prime}\left(r_{0}\right)+\left|f_{2}^{\prime}\left(r_{0}\right)\right|^{p}-\alpha f_{2}\left(r_{0}\right) \\
& =-\frac{n-1}{r_{0}} f_{1}^{\prime}\left(r_{0}\right)-\frac{r_{0}}{2} f_{1}^{\prime}\left(r_{0}\right)+\left|f_{1}^{\prime}\left(r_{0}\right)\right|^{p}-\alpha f_{2}\left(r_{0}\right) \\
& =f_{1}^{\prime \prime}\left(r_{0}\right)+\alpha\left(f_{1}\left(r_{0}\right)-f_{2}\left(r_{0}\right)\right)<f_{1}^{\prime \prime}\left(r_{0}\right) .
\end{aligned}
$$

It contradicts to the definition of $r_{0}$. This lemma is proved.

Proof of Theorem 2.1(ii). By Lemmas 2.4-2.6 and the proofs of Lemmas 2.4 and 2.5 we know that there exist $a_{i}: 0<a_{1} \leq a_{2}<\infty$ such that $\mathcal{A}=$ $\left(0, a_{1}\right), \quad \mathcal{B}=\left(a_{2}, \infty\right), \quad \mathcal{C}=\left[a_{1}, a_{2}\right]$.

For any $a \in \mathcal{C}$, the corresponding orbit satisfies

$$
\lim _{r \rightarrow \infty} \frac{f^{\prime}(r)}{f(r)}=-\infty
$$

To prove (2.3), we define $E(r)=r v(r)+\frac{1}{2} r^{2} f(r), \quad G(r)=r^{2} E(r)-(2 \alpha-$ $n) r^{2} f(r)$. Similar to the proofs of Lemmas 13-15 in [4] we can prove that $\lim _{r \rightarrow \infty} \frac{v(r)}{r f(r)}=-\frac{1}{2}, \quad \lim _{r \rightarrow \infty} \frac{E(r)}{f(r)}=2 \alpha-n, \quad \lim _{r \rightarrow \infty} \frac{G(r)}{f(r)}=4(\alpha-1)(2 \alpha-n)$.

Using the same argument as in the proof of Theorem 2 in [4] it follows that (2.3) holds.

For any $a \in \mathcal{B}$, the corresponding orbit satisfies

$$
\lim _{r \rightarrow \infty} \frac{f^{\prime}(r)}{f(r)}=0 .
$$

Similar to the proof of Theorem 4 in [4] we know that (2.2) holds.

We prove $a_{1}=a_{2}$. If $a_{1}<a_{2}$, by Lemma 2.6 and (2.10) we have that $w^{\prime}+b(r) w=F(r)>0$ for all $r>0$, where $w=f_{1} f_{2}^{\prime}-f_{1}^{\prime} f_{2}$ and $b(r):=$ $\frac{n-1}{r}+\frac{r}{2}+\left|f_{2}^{\prime}\right|^{p-1}$. Therefore,

$$
w(R) \exp \left\{\int_{r_{0}}^{R} b(s) d s\right\}>w(r) \exp \left\{\int_{r_{0}}^{r} b(s) d s\right\} .
$$

Because

$f_{i}(r) \approx k\left(a_{i}\right) r^{2 \alpha-n} \exp \left\{-\frac{r^{2}}{4}\right\}, f_{i}^{\prime}(r) \approx k\left(a_{i}\right) r^{2 \alpha+1-n} \exp \left\{-\frac{r^{2}}{4}\right\}, r \gg 1$,

it follows that

$$
w(r) \exp \left\{\int_{r_{0}}^{r} b(s) d s\right\}<w(R) \exp \left\{\int_{r_{0}}^{R} b(s) d s\right\} \rightarrow 0 \text { as } R \rightarrow \infty .
$$

It is a contradiction. Therefore, $a_{1}=a_{2}:=a^{*}$.

Lemma 2.6 shows that $f(r ; a)$ is strictly increasing and $f^{\prime}(r ; a)$ is strictly decreasing with respect to $a$. The proof of Theorem 2.1 is complete. 


\section{Existence of very singular self-similar solution of (1.2).}

In this section we prove the existence and uniqueness of very singular selfsimilar solution to (1.2). Similar to $\S 2$, we consider (1.7) with initial data

$$
f(0)=b>0, \quad f^{\prime}(0)=0 .
$$

For each $b>0,(1.7),(3.1)$ has a unique solution $f(r ; b)$. If we denote by $(0, R(b))$ the maximal existence interval where $f>0$, then $f^{\prime}<0$ in $(0, R(b))$. The main results of this section read as follows.

Theorem 3.1. Assume that $1<m<p<2$. Then the following conclusions hold:

(i) If $\alpha \leq n \beta$, then $R(b)=\infty$ and $\liminf _{r \rightarrow \infty} r^{\alpha / \beta} f(r ; b)>0$.

(ii) If $\alpha>n \beta$, then there exist one closed set $\mathcal{B}$ and two open sets $\mathcal{A}$ and $\mathcal{C}$ of $(0, \infty)$ satisfying

$\mathcal{A} \cup \mathcal{B} \cup \mathcal{C}=(0, \infty), \quad(b, \infty) \subset \mathcal{A}$ if $b \gg 1$, and $(0, b) \subset \mathcal{C}$ if $0<b \ll 1$, such that the following classification is valid:

(a) If $b \in \mathcal{C}$, then $R(b)<\infty$ and $\left(f^{m}\right)^{\prime}(R(b))<0$.

(b) If $b \in \mathcal{A}$, then $R(b)=\infty, \lim _{r \rightarrow \infty}\left(f(r ; b), f^{\prime}(r ; b)\right)=(0,0)$. And for some $\varphi(b)>0$,

$$
\lim _{r \rightarrow \infty} r^{\alpha / \beta} f(r ; b)=\varphi(b) .
$$

(c) If $b \in \mathcal{B}$, then $R(b)<\infty$ and $f^{\prime}(R(b))=0$. That is, the solution $f(r ; b)$ has compact support, and

$$
f(r)>0 \text { for } 0 \leq r<R(b), \quad f(r)=0 \text { for } r \geq R(b) .
$$

Moreover,

$$
\lim _{r \nearrow R(b)}\left(f^{m-1}(r)\right)^{\prime}=-\frac{(m-1) \beta}{m} R(b) .
$$

Where $\alpha$ and $\beta$ are given in (1.6).

This theorem shows that (1.2) has a very singular self-similar solution if and only if $\alpha>n \beta$.

Remark. (3.2) is the important profile relation which we believe should give the optimal regularity of general solutions with compact support.

The proof of Theorem 3.1 (i) is similar to that of Theorem 2.1 (i), and we omit the details.

In the sequel we assume that $\alpha>n \beta$.

Let $z=f^{m}, \quad a=b^{m}$, we deal with the reduced problem

$$
\left\{\begin{array}{l}
z^{\prime \prime}+\frac{n-1}{r} z^{\prime}+\beta r\left(z^{1 / m}\right)^{\prime}+\alpha z^{1 / m}-m^{-p} z^{p(1-m) / m}\left|z^{\prime}\right|^{p}=0 \quad r>0 \\
z(0)=a>0, \quad z^{\prime}(0)=0, \quad z(r) \geq 0
\end{array}\right.
$$


Here, (1.10) becomes

$$
\lim _{r \rightarrow \infty} r^{\alpha / \beta} z^{1 / m}(r)=0 .
$$

Let $z^{\prime}=v$ then we have

$$
\left\{\begin{array}{l}
z^{\prime}=v \\
v^{\prime}=-\frac{n-1}{r} v-\frac{\beta}{m} r z^{\frac{1}{m}-1} v-\alpha z^{\frac{1}{m}}-m^{-p} z^{p(1-m) / m}|v|^{p-1} v .
\end{array}\right.
$$

Lemma 3.1. Assume that $a>0$ and $z=z(r ; a)$ is the solution of (3.3), $(0, R(a))$ is the maximal existence interval where $z>0$. Then $z^{\prime}(r)<0$ for all $0<r<R(a)$, and

$$
\left|z^{\prime}(r)\right| \leq m \alpha^{1 / p} a^{(1+m p-p) /(m p)} \quad \text { for all } 0 \leq r \leq R(a) .
$$

Proof. It's proof is similar to that of Lemma 2.1 and we omit the details.

Because $1<m<p<2$, it is clear that there exists $\theta$ such that

$$
\max \left\{\frac{1}{m}, \frac{1+m p-p}{m p}\right\}<\theta<\min \left\{1, \frac{2 p-1}{m p}, \frac{m+p-m p}{m(2-p)}\right\} .
$$

For any given $\lambda, \eta>0$, we define

$$
\mathcal{S}_{\lambda, \eta}:=\left\{(z, v): 0<z \leq \eta, \quad-\lambda z^{\theta}<v<0\right\} .
$$

Lemma 3.2. For any given $\lambda, \quad \eta>0$, there exists an $r_{\lambda, \eta}:=\frac{m \alpha}{\beta \lambda} \eta^{1-\theta}+$ $\frac{m \theta \lambda}{\beta} \eta^{\theta-1 / m}$ such that $\mathcal{S}_{\lambda, \eta}$ is positively invariant for $r>r_{\lambda, \eta}$. That is, if $\left(z\left(r_{\lambda, \eta}\right), v\left(r_{\lambda, \eta}\right)\right) \in \mathcal{S}_{\lambda, \eta}$, then the orbit $(z(r), v(r))$ of $(3.5)$ remains in $\mathcal{S}_{\lambda, \eta}$ for all $r \geq r_{\lambda, \eta}$.

Proof. Similar to the proof of Lemma 2.2, we need only to show that the orbit points into $\mathcal{S}_{\lambda, \eta}$ from the parabola

$$
l_{\lambda, \eta}:=\left\{(z, v): 0<z \leq \eta, \quad v=-\lambda z^{\theta}\right\} .
$$

On $l_{\lambda, \eta}$, by $(3.5)$, we have

$$
\begin{aligned}
\frac{v^{\prime}}{\left(z^{\theta}\right)^{\prime}}= & -\frac{n-1}{\theta r} z^{1-\theta}-\frac{\beta}{m \theta} r z^{\frac{1}{m}-\theta}+\frac{\alpha}{\theta \lambda} z^{1-2 \theta+\frac{1}{m}} \\
& -\frac{1}{\theta} m^{-p} z^{1-\theta+p(1-m) / m}|v|^{p-1} \\
< & -\frac{\beta}{m \theta} r z^{\frac{1}{m}-\theta}+\frac{\alpha}{\theta \lambda} z^{1-2 \theta+\frac{1}{m}} \leq-\lambda
\end{aligned}
$$

if

$$
-\frac{\beta}{m \theta} r+\frac{\alpha}{\theta \lambda} z^{1-\theta} \leq-\lambda z^{\theta-\frac{1}{m}}
$$

Since $1 / m<\theta<1$ and $0<z \leq \eta$, it is clear that (3.8) holds if

$$
r \geq r_{\lambda, \eta}:=\frac{m \alpha}{\beta \lambda} \eta^{1-\theta}+\frac{m \theta \lambda}{\beta} \eta^{\theta-1 / m} .
$$


This completes the proof.

Define $\mathcal{S}_{\lambda}:=\left\{(z, v): z>0, \quad-\lambda z^{\theta}<v<0\right\}$. The orbit $(z(r), v(r))$ of (3.5) starting from $(a, 0)$ enters $\mathcal{S}_{\lambda}$ eventually means that there exists an $r_{a}: 0<r_{a}<R(a)$ such that $(z(r), v(r)) \in \mathcal{S}_{\lambda}$ for all $r_{a} \leq r<R(a)$. Set $\mathcal{A}=\left\{a>0\right.$ : the orbit $(z, v)$ starting from $(a, 0)$ enters $\mathcal{S}_{1}$ eventually $\}$, $\mathcal{B}=\left\{a>0: R(a)<\infty, \quad z^{\prime}(R(a))=0\right\}$, $\mathcal{C}=\left\{a>0: R(a)<\infty, \quad z^{\prime}(R(a))<0\right\}$.

Remark. For any $a \in \mathcal{A}$, the corresponding solution $z(r ; a)$ satisfies $z^{\prime}+$ $z^{\theta}>0$ when $r<R(a)$ and close to $R(a)$. This implies $R(a)=\infty$. Therefore, $\mathcal{A} \cup \mathcal{B} \cup \mathcal{C}=(0, \infty)$. It is obvious that $\mathcal{A}, \mathcal{B}$ and $\mathcal{C}$ do not intersect with each other.

Lemma 3.3. The set $\mathcal{C}$ is non-empty and open. Moreover, $(0, b) \subset \mathcal{C}$ if $0<b \ll 1$.

Proof. We choose $a=\epsilon>0$ small and let $w_{\epsilon}(t)=\varepsilon^{-1} z(r ; \varepsilon), t=r \varepsilon^{(1-m) /(2 m)}$. For simplicity we replace $t$ by $r$. Then $w_{\varepsilon}$ satisfies

$$
\left\{\begin{array}{l}
w_{\varepsilon}^{\prime \prime}+\frac{n-1}{r} w_{\varepsilon}^{\prime}+\beta r\left(w_{\varepsilon}^{1 / m}\right)^{\prime}+\alpha w_{\varepsilon}^{1 / m} \\
\quad+m^{-p} \varepsilon^{(3 p-m p-2) /(2 m)} w_{\varepsilon}^{p(1-m) / m}\left|w_{\varepsilon}^{\prime}\right|^{p-1} w_{\varepsilon}^{\prime}=0, \\
w_{\varepsilon}(0)=1, \quad w_{\varepsilon}^{\prime}(0)=0 .
\end{array}\right.
$$

Let $E\left(w_{\varepsilon}\right)=\frac{2 \alpha}{1+1 / m} w_{\varepsilon}^{(1+m) / m}+\left(w_{\varepsilon}^{\prime}\right)^{2}$, then $\frac{d}{d r} E\left(w_{\varepsilon}\right) \leq 0$. Therefore, $E\left(w_{\varepsilon}\right) \leq$ $2 m \alpha /(1+m)$ for all $\varepsilon>0$, both $w_{\varepsilon}$ and $w_{\varepsilon}^{\prime}$ are uniformly bounded with respect to $r \geq 0$ and $\varepsilon>0$. Denote by $\left(0, R_{\varepsilon}\right)$ the maximal existence interval where $w_{\varepsilon}>0$, then $w_{\varepsilon}^{\prime}(r)<0$ in $\left(0, R_{\varepsilon}\right)$.

We first consider the reduced problem $(\varepsilon=0)$

$$
\left\{\begin{array}{l}
w^{\prime \prime}+\frac{n-1}{r} w^{\prime}+\beta r\left(w^{1 / m}\right)^{\prime}+\alpha w^{1 / m}=0, \\
w(0)=1, \quad w^{\prime}(0)=0 .
\end{array}\right.
$$

It is easy to show that there exists an $r_{0}: 0<r_{0}<\infty$ such that $w\left(r_{0}\right)=$ $0, w^{\prime}\left(r_{0}\right)<0$ and $w(r)>0$ for all $0 \leq r<r_{0}$.

We will prove that when $\varepsilon$ is small then the solution $w_{\varepsilon}$ of (3.9) has the same properties as $w$. To this aim, let $\eta_{0}>0$ be such that

$$
\eta_{0}+\frac{(m-1)}{8 m} r_{0} w^{\prime}\left(r_{0}\right)<0,
$$

$$
\begin{aligned}
\frac{n-1}{r_{0}} \eta_{0} & +\beta r_{0} \eta_{0}^{1 / m} \\
& +\frac{m^{1-p}}{m+p-m p}\left(\frac{2 m \alpha}{1+m}\right)^{(p-1) / 2} \eta_{0}^{(m+p-m p) / m}<-\frac{w^{\prime}\left(r_{0}\right)}{4} .
\end{aligned}
$$


Choose $r_{1}: 0<r_{0}-r_{1} \ll 1$ such that $w\left(r_{1}\right)<\eta_{0}$ and $w^{\prime}\left(r_{1}\right)<w^{\prime}\left(r_{0}\right) / 2$. By the continuous dependence of solution on the parameter $\varepsilon$ we have that if $\varepsilon \ll 1$, then the solution $w_{\varepsilon}$ of (3.9) satisfies

$$
R_{\varepsilon}>r_{1}, \quad w_{\varepsilon}\left(r_{1}\right):=\eta<\eta_{0}, \quad w_{\varepsilon}^{\prime}\left(r_{1}\right)<w^{\prime}\left(r_{0}\right) / 2 .
$$

Since $\left|w_{\varepsilon}^{\prime}(r)\right| \leq[2 m \alpha /(1+m)]^{1 / 2}$, it follows that for $r>r_{1}$,

$$
\begin{aligned}
w_{\varepsilon}^{\prime \prime} \leq-\frac{n-1}{r} w_{\varepsilon}^{\prime}- & \beta r\left(w_{\varepsilon}^{1 / m}\right)^{\prime}-\alpha w_{\varepsilon}^{1 / m} \\
& -m^{-p} \varepsilon^{(3 p-m p-2) /(2 m)}\left(\frac{2 m \alpha}{1+m}\right)^{(p-1) / 2} w_{\varepsilon}^{p(1-m) / m} w_{\varepsilon}^{\prime} .
\end{aligned}
$$

Integrating this inequality from $r_{1}$ to $r$ and using (3.11), (3.12) we have,

$$
\begin{aligned}
w_{\varepsilon}^{\prime}+\beta r w_{\varepsilon}^{1 / m} \leq & w_{\varepsilon}^{\prime}\left(r_{1}\right)+\frac{n-1}{r_{1}} \eta+\beta r_{1} \eta^{1 / m}+\beta \eta^{1 / m}\left(r-r_{1}\right) \\
& +\frac{m^{1-p}}{m+p-m p}\left(\frac{2 m \alpha}{1+m}\right)^{(p-1) / 2} \varepsilon^{(3 p-m p-2) /(2 m)} \eta^{(m+p-m p) / m} \\
\leq & \frac{1}{2} w^{\prime}\left(r_{0}\right)+\frac{n-1}{r_{0}} \eta_{0}+\beta r_{0} \eta_{0}^{1 / m}+\beta \eta^{1 / m}\left(r-r_{1}\right) \\
& +\frac{m^{1-p}}{m+p-m p}\left(\frac{2 m \alpha}{1+m}\right)^{(p-1) / 2} \eta_{0}^{(m+p-m p) / m} \\
< & \frac{1}{4} w^{\prime}\left(r_{0}\right)+\beta \eta^{1 / m}\left(r-r_{1}\right) \leq 0
\end{aligned}
$$

if

$$
r \leq r_{2}:=r_{1}-\frac{1}{4 \beta} \eta^{-1 / m} w^{\prime}\left(r_{0}\right) .
$$

Integrating (3.13) from $r_{1}$ to $r_{2}$ and by (3.10) we get

$$
\begin{aligned}
w_{\varepsilon}^{(m-1) / m}\left(r_{2}\right) & <\eta^{(m-1) / m}-\frac{\beta(m-1)}{2 m}\left(r_{2}^{2}-r_{1}^{2}\right) \\
& <\eta^{-1 / m}\left[\eta+\frac{(m-1)}{8 m} r_{0} w^{\prime}\left(r_{0}\right)\right] \\
& <\eta^{-1 / m}\left[\eta_{0}+\frac{(m-1)}{8 m} r_{0} w^{\prime}\left(r_{0}\right)\right]<0 .
\end{aligned}
$$

This shows that $R_{\varepsilon}<r_{2}$ and $w_{\varepsilon}^{\prime}+\beta r w_{\varepsilon}^{1 / m}<0$ for all $r_{1}<r \leq R_{\varepsilon}$. Therefore, $w_{\varepsilon}^{\prime}\left(R_{\varepsilon}\right)<0$. And consequently, $a=\varepsilon \in \mathcal{C}$.

It can be seen from the above proof that for a solution $z$ to (3.3), if there exists $r_{1}>0$ such that $z\left(r_{1}\right) \ll 1$ and $\left|z^{\prime}\left(r_{1}\right)\right|$ is not too small, then the solution will reach zero at a finite $r=R$ and $z^{\prime}(R)<0$. Hence $\mathcal{C}$ is open. Lemma 3.3 is proved. 
Lemma 3.4. For any given $a>0$. If the corresponding $R(a)=\infty$, then there exists a limit $\lim _{r \rightarrow \infty} r^{\alpha / \beta} z^{1 / m}(r ; a)=k(a)$ and $k(a)>0$.

Proof.

Step 1. First, as in the case of semilinear case, it can be shown following the argument of Lemmas 5-7 in [4] that $z(r) \rightarrow 0$ as $r \rightarrow \infty$. Since $z(r)>0$, $z^{\prime}(r)<0$ for all $r>0$ and $z(r) \rightarrow 0$ as $r \rightarrow \infty$, it is impossible that $z^{\prime \prime}(r)<0$ for all $r \gg 1$. Differentiating Equation (3.3), it is easy to see that $z^{\prime \prime}(r)>0$ for $r \gg 1$, in consequence, by (3.3), $\alpha z^{1 / m}+\beta r\left(z^{1 / m}\right)^{\prime}+\frac{m(n-1)}{r} z^{(m-1) / m}\left(z^{1 / m}\right)^{\prime}-\left|\left(z^{1 / m}\right)^{\prime}\right|^{p}<0, \quad \forall r \gg 1$. For any given $\varepsilon>0$, since $p>m>1$ and $\left(z, z^{\prime}\right) \rightarrow(0,0)$ as $r \rightarrow \infty$, it follows that

$$
\alpha z^{1 / m}+(\beta+\varepsilon) r\left(z^{1 / m}\right)^{\prime}<0, \quad \forall r \gg 1,
$$

and consequently,

$$
z(r) \leq C r^{-m \alpha /(\beta+\varepsilon)}, \quad \forall r \gg 1 .
$$

Step 2. Chosen $\mu>m \alpha / \beta$ and define $h(r)=\mu z(r ; a)+r z^{\prime}(r ; a)$. We claim that $h(r)$ does not change signs for $r \gg 1$. In fact, if $h\left(r_{0}\right)=0$, then by using (3.3) we have

$$
h^{\prime}\left(r_{0}\right)=-\frac{\mu(\mu+2-n)}{r_{0}} z+(\mu \beta / m-\alpha) r_{0} z^{1 / m}+r_{0}^{1-p} m^{-p} \mu^{p} z^{p / m}>0
$$

provided that $r_{0}$ satisfies:

$$
z^{(m-1) / m}\left(r_{0} ; a\right)<\frac{\mu \beta / m-\alpha}{\mu(\mu+2-n)} r_{0}^{2} \quad(\alpha>n \beta \Rightarrow \mu>n) .
$$

Hence, $h(r)>0$ for $r>r_{0}$. Therefore,

$$
h(r)<0 \quad \text { for all } r \gg 1 \text {; or } \quad h(r)>0 \text { for all } r \gg 1 .
$$

If $h(r)=\mu z+r z^{\prime}<0$ for all $r \gg 1$, then $-r z^{\prime} / z>\mu$, and consequently

$$
\begin{aligned}
z^{\prime \prime} & =-\frac{n-1}{r} z^{\prime}+z^{1 / m}\left(-\alpha-\beta r z^{\prime} /(m z)\right)+m^{-p} z^{p(1-m) / m}\left|z^{\prime}\right|^{p} \\
& >z^{1 / m}(\beta \mu / m-\alpha) \triangleq \delta z^{1 / m}, \quad \delta>0, \quad r \gg 1 .
\end{aligned}
$$

Multiplying (3.15) by $z^{\prime}$ and integrating the results from $r$ to $\infty$ we have

$$
\left(z^{\prime}\right)^{2} \geq[m \delta /(1+m)] z^{(1+m) / m}, \quad \text { i.e., } \quad-z^{\prime} \geq C z^{(1+m) /(2 m)}, \quad r \gg 1 .
$$

Since $m>1$, an integration of the last inequality yields that $z\left(r_{0}\right)=0$ for some $r_{0}<\infty$. It is a contradiction. Therefore, $h(r)>0$ for all $r \gg 1$.

By using (3.14) we have

$$
\left|z^{\prime}\right|=-z^{\prime} \leq \mu r^{-1} z=O\left(r^{-1-m \alpha /(\beta+\varepsilon)}\right) .
$$


Multiplying (3.3) by $r^{(\alpha-\beta) / \beta}$ and an integration from 0 to $r$ yields

$$
\begin{aligned}
& z^{\prime} r^{(\alpha-\beta) / \beta}+\beta r^{\alpha / \beta} z^{1 / m} \\
& =\left(\frac{\alpha}{\beta}-n\right) \int_{0}^{r} z^{\prime} s^{\frac{\alpha}{\beta}-2} d s+m^{-p} \int_{0}^{r} z^{\frac{p(1-m)}{m}}\left|z^{\prime}\right|^{p} s^{\frac{\alpha}{\beta}-1} d s .
\end{aligned}
$$

By (3.16) it follows that two integrands of the right hand side of (3.17) converge and $\lim _{r \rightarrow \infty} z^{\prime} r^{(\alpha-\beta) / \beta}=0$. Consequently, the limit

$$
\lim _{r \rightarrow \infty} r^{\alpha / \beta} z^{1 / m}=k(a)
$$

exists. It is obvious that $k(a) \geq 0$.

Now we show tht $k(a)>0$. Assuming on the contrary that $k(a)=0$. Multiplying (3.3) by $r^{(\alpha-\beta) / \beta}$ and an integration from $r$ to $\infty$ yields, in view of (3.16),

$$
\begin{aligned}
& z^{\prime} r^{(\alpha-\beta) / \beta}+\beta r^{\alpha / \beta} z^{1 / m} \\
& =\left(n-\frac{\alpha}{\beta}\right) \int_{r}^{\infty} z^{\prime} s^{\frac{\alpha}{\beta}-2} d s-m^{-p} \int_{r}^{\infty} z^{\frac{p(1-m)}{m}}\left|z^{\prime}\right|^{p} s^{\frac{\alpha}{\beta}-1} d s .
\end{aligned}
$$

Consequently, $z r^{M} \rightarrow 0$ as $r \rightarrow \infty$ for any $M>0$.

On the other hand, since $h(r)>0$ for $r \gg 1$, it follows that $z(r) \geq C r^{-\mu}$ for $r \gg 1$. We get a contradiction. Therefore, $k(a)>0$.

Lemma 3.5. The set $\mathcal{A}$ is non-empty and open. Moreover, for any $a \in \mathcal{A}$, there exists a limit $\lim _{r \rightarrow \infty} r^{\alpha / \beta} z^{1 / m}(r ; a)=k(a)$ and $k(a)>0$.

Proof. Using (3.6) and the special choice of $\theta$ (see (3.7)), by following the proof of Lemma 2.5 we can show that if $a$ is large, it is in $\mathcal{A}$. Since $1 / m<$ $\theta<1$, the number $r_{1, \eta}:=\frac{m \alpha}{\beta} \eta^{1-\theta}+\frac{m \theta}{\beta} \eta^{\theta-1 / m} \rightarrow 0$ as $\eta \searrow 0$. Lemma 3.2 shows that $\mathcal{A}$ is open.

The last conclusion is a corollary of Lemma 3.4.

Lemma 3.6. The set $\mathcal{B}$ is non-empty. For any $a \in \mathcal{B}$, the corresponding solution $z(r ; a)$ satisfies the following interface relation:

$$
\lim _{r \nearrow R(a)}\left\{z^{\prime} / z^{1 / m}\right\}=-\beta R(a) .
$$

Proof. From Lemmas 3.3 and 3.5 we know that $\mathcal{B}$ is non-empty.

For simplicity we denote $R=R(a)$. Putting Equation (3.3) into divergence form and integrating the results from $r$ to $R$, we get

$$
\begin{aligned}
r^{n-1} z^{\prime}= & -\beta r^{n} z^{1 / m}+(\alpha-n \beta) \int_{r}^{R} z^{1 / m} s^{n-1} d s \\
& -m^{-p} \int_{r}^{R} z^{p(1-m) / m}\left|z^{\prime}\right|^{p} s^{n-1} d s .
\end{aligned}
$$


Dividing (3.18) by $z^{1 / m}$ and putting $r \rightarrow R$ yields

$$
R^{n-1} \lim _{r \rightarrow R} \frac{z^{\prime}}{z^{1 / m}}=-\beta R^{n}-m^{-p} \lim _{r \rightarrow R} z^{-1 / m} \int_{r}^{R} z^{p(1-m) / m}\left|z^{\prime}\right|^{p} s^{n-1} d s .
$$

By using L'Hospital's rule we get

$$
\lim _{r \rightarrow R} z^{-1 / m} \int_{r}^{R} z^{p(1-m) / m}\left|z^{\prime}\right|^{p} s^{n-1}=m R^{n-1} \lim _{r \rightarrow R}\left(\left|z^{\prime}\right| / z^{(m-1) / m}\right)^{p-1} d s
$$

if the limit of the right hand side of (3.20) exists.

In the following we will prove that

$$
\lim _{r \rightarrow R}\left\{\left|z^{\prime}\right| / z^{(m-1) / m}\right\}=0 .
$$

Dividing (3.18) by $z^{(m-1) / m}$ and putting $r \rightarrow R$, note that $1 / m>(m-$ $1) / m$, we have

$$
R^{n-1} \lim _{r \rightarrow R} \frac{z^{\prime}}{z^{(m-1) / m}}=-\frac{m^{1-p}}{m-1} R^{n-1} \lim _{r \rightarrow R} z^{(p+1-m p) / m}\left|z^{\prime}\right|^{p-1}
$$

if the limit of the right hand side of (3.22) exists.

Choose $\sigma_{1}=(p+m-m p) / m$, then $\sigma_{1}<1 / m$. Similar to (3.22) we have

$$
R^{n-1} \lim _{r \rightarrow R} \frac{z^{\prime}}{z^{\sigma_{1}}}=-\frac{m^{-p}}{\sigma_{1}} R^{n-1} \lim _{r \rightarrow R}\left|z^{\prime}\right|^{p-1}=0 .
$$

Case 1 . If $p+1 \geq m p$, then $\sigma_{1} \geq(m-1) / m$, and consequently,

$$
\lim _{r \rightarrow R}\left\{\left|z^{\prime}\right| / z^{(m-1) / m}\right\} \leq \lim _{r \rightarrow R}\left\{\left|z^{\prime}\right| / z^{\sigma_{1}}\right\}=0,
$$

i.e., (3.21) holds.

Case 2. If $p+1<m p$. We write

$$
z^{(p+1-m p) / m}\left|z^{\prime}\right|^{p-1}=\left(\frac{\left|z^{\prime}\right|}{z^{(m p-p-1) /(m p-m)}}\right)^{p-1},
$$

and define a sequence

$$
\sigma_{l+1}=(p-1) \sigma_{l}+1-p+p / m, \quad l=1,2, \cdots .
$$

We first discuss the properties of $\sigma_{l}$. If $\sigma_{l}<(m p-p-1) /(m p-m)$ for some $l$, then

$$
\sigma_{l}<\frac{m+p-m p}{m(2-p)}, \quad \text { and } \quad \frac{m p-p-1}{m p-m}<\frac{m+p-m p}{m(2-p)}
$$

because $m<2$. The first inequality of (3.26) implies $\sigma_{l+1}>\sigma_{l}$. We assert that there exists $l$ such that $\sigma_{l} \geq(m p-p-1) /(m p-m)$. Otherwise, $\sigma_{l} \rightarrow \sigma_{0}$ as $l \rightarrow \infty$ for some $\sigma_{0}>0$ and $\sigma_{0} \leq(m p-p-1) /(m p-m)$. Letting $l \rightarrow \infty$ 
in (3.25) yields $\sigma_{0}=(m+p-m p) /(2 m-m p)$. It contradicts with the second inequality of (3.26).

We assume that $l_{0} \geq 1$ is the first one such that $\sigma_{l_{0}} \geq(m p-p-1) /(m p-$ $m)$. If $l_{0}=1$, then $\lim _{r \rightarrow R} z^{(p+1-m p) / m}\left|z^{\prime}\right|^{p-1} \leq \lim _{r \rightarrow R}\left(\left|z^{\prime}\right| / z^{\sigma_{1}}\right)^{p-1}=0$ by (3.23). Hence, (3.21) holds in view of (3.22). If $l_{0}>1$, we have

$$
\begin{aligned}
\sigma_{l+1} & =(p-1) \sigma_{l}+1-p+p / m<(m p-p-1) / m+1-p+p / m \\
& =1-1 / m<1 / m, \quad \forall 1 \leq l<l_{0}
\end{aligned}
$$

because $\sigma_{l}<(m p-p-1) /(m p-m)$. Moreover, $\sigma_{l+1}>\sigma_{l}>\cdots>\sigma_{1}=$ $(m+p-m p) / m$ for all $1 \leq l \leq \sigma_{l_{0}}-1$. Dividing (3.18) by $z^{\sigma_{l+1}}, 1 \leq l<l_{0}$, we have

$$
\begin{aligned}
& R^{n-1} \lim _{r \rightarrow R} \frac{z^{\prime}}{z^{\sigma_{l+1}}} \\
& =-m^{-p} \lim _{r \rightarrow R} z^{-\sigma_{l+1}} \int_{r}^{R} z^{p(1-m) / m}\left|z^{\prime}\right|^{p} s^{n-1} d s, \quad l=1,2, \ldots, l_{0}-1,
\end{aligned}
$$

and

$$
\begin{aligned}
& \lim _{r \rightarrow R} z^{-\sigma_{l+1}} \int_{r}^{R} z^{p(1-m) / m}\left|z^{\prime}\right|^{p} s^{n-1} d s \\
& =\frac{R^{n-1}}{\sigma_{l+1}} \lim _{r \rightarrow R}\left(\left|z^{\prime}\right| / z^{\sigma_{l}}\right)^{p-1}, \quad l=1,2, \ldots, l_{0}-1
\end{aligned}
$$

provided that the limit of the right hand side of (3.28) exists. Using (3.23) we have that the limit of the left hand side of (3.28) equals zero for $l=1$. Step by step, by repeatedly using (3.27) and (3.28) it follows that the conclusion holds for $l=l_{0}-1$. That is,

$$
R^{n-1} \lim _{r \rightarrow R} \frac{z^{\prime}}{z^{\sigma_{l_{0}}}}=-m^{-p} \lim _{r \rightarrow R} z^{-\sigma_{l_{0}}} \int_{r}^{R} z^{p(1-m) / m}\left|z^{\prime}\right|^{p} s^{n-1} d s=0 .
$$

Because $\sigma_{l_{0}} \geq(m p-p-1) /(m p-m)$, by use of (3.22) it follows that (3.21) holds.

From (3.19)-(3.21) we know that Lemma 3.6 holds.

Proof of Theorem 3.1 (ii). Denote $\varphi(b)=k\left(b^{m}\right)$. By Lemmas 3.3, 3.5 and 3.6 we know that Theorem 3.1 (ii) holds.

\section{References}

[1] M. Ben-Artzi and H. Koch, Decay of mass for a semilinear parabolic equation, Comm. Partial Diff. Equns., 24(5-6) (1999), 869-881, MR 2000a:35098, Zbl 931.35066.

[2] S. Benachour and Ph. Laurencot, Solutions tres singulieres d'une equation parabolique non lineaire avec absorption, C.R. Acad. Sci. Paris, 328 (1999), 215-220, MR 99k:35080, Zbl 926.35057. 
[3] H. Brezis and A. Friedman, Nonlinear parabolic equations involving measures as initial conditions, J. Math. Pures Appl., 62 (1983), 73-97, MR 84g:35093, Zbl 527.35043.

[4] H. Brezis, L.A. Peletier and D. Terman, A very singular solution of the heat equation with absorption, Arch. Rat. Mech. Anal., 96 (1986), 185-209, MR 87i:35100, Zbl 627.35046.

[5] X. Chen, Y. Qi and M. Wang, Self-similar very singular solutions of the parabolic p-Laplacian, preprint.

[6] _ Existence and uniqueness of singular solutions of a fast diffusion porous medium equation, preprint.

[7] J.I. Diaz and J.E. Saa, Uniqueness of very singular self-similar solution of a quasilinear degenerate parabolic equation with absorption, Publ. Math., 36 (1992), 19-38, MR 93g:35079, Zbl 794.35091.

[8] E. DiBenedetto and M. Herrero, Non-negative solutions of the evolution p-Laplacian equation. Initial traces and Cauchy problem when $1<p<2$, Arch. Rat. Mech. Anal., 111 (1990), 225-290, MR 92g:35088, Zbl 726.35066.

[9] M. Escobedo and O. Kavian, Variational problems related to self-similar solutions of the heat equation, Nonlinear Analysis TMA, 11 (1987), 1103-1133, MR 90a:35128, Zbl 639.35038

[10] B. Gilding, M. Guedda and R. Kersner, The Cauchy problem for $u_{t}=\triangle u+|\nabla u|^{q}$, preprint, 1998.

[11] S. Kamin and L.A. Peletier, Singular solutions of the heat equation with absorption, Proc. Amer. Math. Soc., 95 (1985), 205-210, MR 87b:35090, Zbl 607.35046.

[12] S. Kamin, L.A. Peletier and J. Vasquez, Classification of singular solutions of a nonlinear heat equation, Duke Math. J., 58 (1989), 601-615, MR 91g:35137, Zbl 701.35083.

[13] S. Kamin and J. Vasquez, Singular solutions of some nonlinear parabolic equations, J. Analyse Math., 59 (1992), 51-74, MR 94e:35079, Zbl 802.35066.

[14] S. Kamin and L. Veron, Existence and uniqueness of the very singular solution for the porous media equation with absorption, J. Analyse Math., 51 (1988), 245-258, MR 90f:35097, Zbl 673.35044.

[15] M. Kardar, G. Parisi and Y.C. Zhang, Dynamic scaling of growing interfaces, Phys. Rev. Lett., 56(9) (1986), 889-892.

[16] J. Krug and H. Spohn, Universality classes for deterministic surface growth, Phys. Rev. A, 38(8) (1988), 4271-4283, MR 89i:82117.

[17] L.A. Peletier and H.C. Serafini, A very singular solution and other self-similar solutions of the heat equation with convection, Nonlinear Analysis, TMA, 24(1) (1995), 29-49, MR 95k:35093, Zbl 824.35058.

[18] L.A. Peletier and D. Terman, A very singular solution of the porous media equation with absorption, J. Diff. Eqns., 65 (1986), 396-410, MR 88b:35090, Zbl 617.35115.

[19] L.A. Peletier and Junyu Wang, A very singular solution of a quasilinear degenerate diffusion equation with absorption, Trans. Amer. Math. Soc., 307 (1988), 813-826, MR 89e:35081, Zbl 696.35094.

[20] L.A. Peletier and J.N. Zhao, Source-type solutions of the porous media equation with absorption: The fast diffusion case, Nonlinear Analysis, TMA, 14 (1990), 107-121, MR 91k:35140, Zbl 702.35134. 
Received September 29, 1999 and revised September 4, 2000. The first author was partially supported by HK RGC grant HKUST630/95P. The second author is grateful to the support of PRC grants NSFC-19771015, 19831060 and HK RGC grant HKUST630/95P.

Department of Mathematics

Hong Kong University of Science \& TeChnology

HONG KONG

E-mail address: maqi@uxmail.ust.hk

Department of Applied Mathematics

SOUTHEAST UNIVERSITY

NANJING 210018

P. R. China

E-mail address: mxwang@seu.edu.cn 